\title{
Supplementary Material: Self-Supervised Adversarial Learning for Cross-Modal Retrieval
}

\author{
Yangchao Wang* \\ Shiyuan $\mathrm{He}^{*}$ \\ yancyycwang@outlook.com \\ shiyuanhe.david@gmail.com \\ Center for Future Media and School \\ of Computer Science and Engineering, \\ University of Electronic Science and \\ Technology of China
}

\author{
Xing $\mathrm{Xu}$ \\ Center for Future Media and School \\ of Computer Science and Engineering, \\ University of Electronic Science and \\ Technology of China \\ xing.xu@uestc.edu.cn
}

\author{
Yang Yang ${ }^{\dagger}$ \\ Center for Future Media and School \\ of Computer Science and Engineering, \\ University of Electronic Science and \\ Technology of China \\ Institute of Electronic and \\ Information Engineering of UESTC in \\ Guangdong \\ dlyyang@gmail.com
}

\author{
Jingjing Li \\ Center for Future Media and School \\ of Computer Science and Engineering, \\ University of Electronic Science and \\ Technology of China \\ lijin117@yeah.net
}

\section{ACM Reference Format:}

Yangchao Wang, Shiyuan He, Xing Xu, Yang Yang, Jingjing Li, and Heng Tao Shen. 2021. Supplementary Material: Self-Supervised Adversarial Learning for Cross-Modal Retrieval. In ACM Multimedia Asia (MMAsia '20), March 7-9, 2021, Virtual Event, Singapore. ACM, New York, NY, USA, 3 pages. https://doi.org/10.1145/3444685.3446269

This supplementary material provides the omitted contents in the main paper.

- Section 1 introduces cross-modal GAN studies in detail.

- Section 2 elaborates the multi-task objective loss with selfsupervision.

- Section 3 presents experimental settings of three datasets.

- Section 4 detailed introduces the implementation details.

- Section 5 desplays the experimental visualisation of the learned representation.

- Section 6 shows the parameter analysis of our experiment.

\section{CROSS-MODAL GAN STUDIES}

Several cross-modal GAN studies $[3,11,12]$ have also been proposed for cross-modal retrieval, in which the strong ability of GAN was

\footnotetext{
${ }^{*}$ Both authors contributed equally to this research.

${ }^{\dagger}$ Corresponding author.
}

Permission to make digital or hard copies of all or part of this work for personal or classroom use is granted without fee provided that copies are not made or distributed for profit or commercial advantage and that copies bear this notice and the full citation on the first page. Copyrights for components of this work owned by others than ACM must be honored. Abstracting with credit is permitted. To copy otherwise, or republish, to post on servers or to redistribute to lists, requires prior specific permission and/or a fee. Request permissions from permissions@acm.org.

MMAsia '20, March 7-9, 2021, Virtual Event, Singapore

(c) 2021 Association for Computing Machinery.

ACM ISBN 978-1-4503-8308-0/21/03 . \$ \$15.00

https://doi.org/10.1145/3444685.3446269
Heng Tao Shen

Center for Future Media and School

of Computer Science and Engineering,

University of Electronic Science and

Technology of China

shenhengtao@hotmail.com

utilized to model heterogeneous data distribution and learn discriminative common representations of different modalities. Among these methods, (ACMR) [11] utilized adversarial learning approach to seeking an effective common subspace. The modality-invariant representations were generated by playing a minimax game between a feature projector and a modality classifier. $\mathrm{Gu}$ [4] incorporated generative processes into the cross-modal feature embedding, through which not only the global abstract features but also the local grounded features were learned. Peng [9] proposed Crossmodal Generative Adversarial Networks (CM-GANs) to explore inter-modality and intra-modality correlation simultaneously in generative and discriminative models. Moreover, Zhu [12] proposed Recipe Retrieval Generative Adversarial Network (R2GAN) to learn a common space for recipe and food.

\section{MULTI-TASK OBJECTIVE LOSS WITH SELF-SUPERVISION}

Given a modified instance $\widetilde{x}=f(x)$, self-supervised models were expected to predict which transformation $f$ was applied. Training a multi-task learning framework which optimizes two losses of the primary and self-supervised tasks while sharing the feature space $[1,2,5]$ is the common approach of utilizing self-supervised labels for another task. In a fully supervised setting, the multi-task objective loss with self-supervision can be formulated as follows:

$$
\begin{aligned}
\mathcal{L}(x, y ; \theta, u, v) & =\frac{1}{M} \sum_{j=1}^{M} \mathcal{L}_{C E}\left(\delta\left(f\left(\tilde{x}_{j} ; \theta\right) ; u\right), y\right) \\
& +\mathcal{L}_{C E}\left(\delta\left(f\left(\tilde{x}_{j} ; \theta\right) ; v\right), j\right),
\end{aligned}
$$

where $\left\{f_{j}\right\}_{j=1}^{M}$ is a set of pre-defined transformations, $M$ is the number of self-supervised labels, $\delta(f(\cdot ; \theta) ; u)$ is primary classifier, $\delta(\cdot ; v)$ is the classifier for self-supervision and $\mathcal{L}_{C E}$ is the crossentropy loss function. 


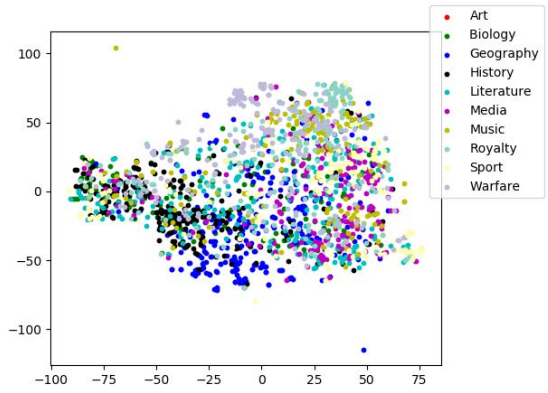

(a)

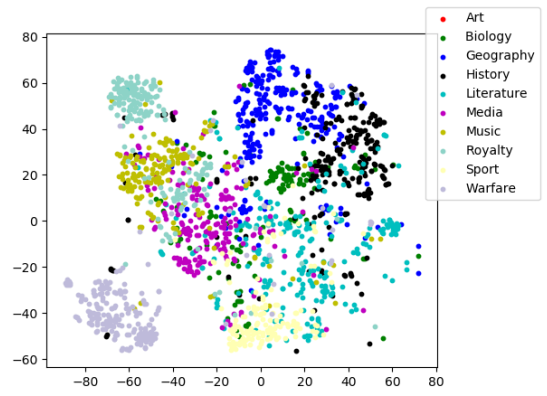

(b)

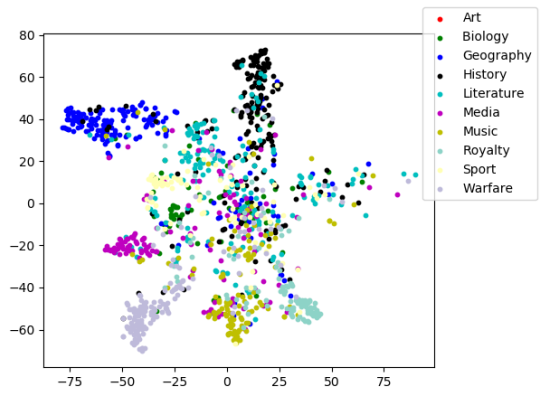

(c)

Figure 1: t-SNE visualization for original features and learned embeddings on Wikipedia dataset. (a)(b) are the original visual and textual features respectively, (c) is mixed embeddings of our SSAL respectively.

\section{DATASETS}

The Wikipedia dataset [10] is the most widely used dataset for crossmodal retrieval, which based on "featured articles" in Wikipedia. The dataset consists of 2,866 image-text pairs, where each pair consists of an image and the corresponding complete text article annotated with a label from 10 semantic classes.

Mirflickr-25k [6] contains 25,000 instances collected from Flickr. Each image is labeled with its associated textual tags. Here, we follow the experimental protocols given in DCMH [7]. In total, 20,015 data points have been selected for our experiment.

MS-COCO [8]: contains 123,287 images and their annotated sentences with their annotations categorized into 80 classes. After pruning images without category information, MSCOCO consists of 60000 training images and 10000 test images, each of which is associated with five sentences.

\section{IMPLEMENTATION DETAILS}

Embedding Generative Network: We built ImgNet and TxtNet with two separate one-layer feed-forward neural networks and a one-layer shared-weights feed-forward neural network, all neural networks are activated by ReLU function. The separate layers are used to project an original feature into an intermediate embedding of size 2048. The shared layers further project the intermediate embedding into an embedding in the common subspace of size 1024 The shared classifier is a two-layer feed-forward neural network, where the mid-layer is activated by ReLU function and the last layer is activated by softmax function.

Self-supervised Adversarial Networks: Adversarial Networks contains two modules to detect the rotation angle and identify the modal category. We built the discriminator networks using a onelayer shared-weights feed-forward neural network activated by Leaky ReLu function and two separate one-layer feed-forward neural networks activated by softmax function. The shared layer first project given embedding of size 1024 into an intermediate embedding of size 512 and the separate layers followed softmax functions further project it into two tensors of size 4 and 2, which are used to predict rotation angles and modalities respectively.

The batch size is set to 128 for all datasets and the hyperparameters are tuned using grid search, which means change one of the hyperparameters 10 times per step and fix the others. Since the proposed SSAL use rotated images, the image and text feature used in our experiments are not the same with that used in other works. For a fair comparison, we re-evaluate the referred methods with the open sourced codes. In addition, SSAL is implemented via Pytorch and is trained on a server with two NVIDIA TITAN X GPUs.

\section{VISUALISATION OF THE LEARNED REPRESENTATION}

To illustrate that our proposed SSAL is able to learn more reliable embeddings than that of previous approaches, we visualize the embeddings of our SSAL on Wikipedia dataset, as shown in the left two columns of Fig. 1. We can see that the distributions of the images and texts are different in the Wikipedia dataset. By comparing Fig. 1(a)(b) and Fig.1(c), it can be observed that our method gets feature representations with more semantic distinction which well separates the mixed representations into several semantically discriminative clusters and can be distinguished easily in the common subspace. In addition, Fig.1(c) also shows that the subspace constructed by our method maps text and image features with the same category information together, making them difficult to distinguish and easy to facilitate cross-modal retrieval.

\section{PARAMETER ANALYSIS}

To investigate the impact of the parameters $\beta, \gamma, \eta$ and $\lambda$ mentioned in main body, we analyze the performance of our SSAL with different parameter values on the test set of the Wikipedia dataset, as shown in Fig. 2. We set the range of parameters as $\{0.001,0.01,0.1$, $1,10,100\}$ and divide these four hyperparameters into two groups according to the adversarial training process and the evaluation is conducted by changing a set of parameters (.e.g, $\beta, \gamma$ ) while fixing the other (.e.g, $\eta, \lambda$ ). From Fig. 2(a), we can observe that SSAL performs well when $\beta$ is in the range of $[0.1,1], \gamma$ is in the range of $[0.01,0.1]$ on Wikipedia. And Fig. 2(b) shows that when $\eta$ is in the range of $[0.001,0.01]$ and $\lambda$ is in the range of $[0.1,1]$, the proposed method achieves the best accuracy. The figure shows that the selected values of hyperparameters help with the overall training optimization process and prevent overfitting so that the proposed method can obtain better results on the test dataset. In addition, we can also see that adversarial learning and reconstruction learning with too much weight will lead to a decrease in performance. 


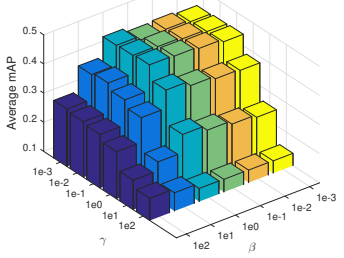

(a)

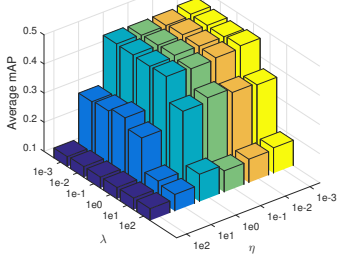

(b)
Figure 2: The Average mAP scores of SSAL versus different values of $\beta, \gamma, \eta$ and $\lambda$ on Wikipedia dataset.

\section{REFERENCES}

[1] Lucas Beyer, Xiaohua Zhai, Avital Oliver, and Alexander Kolesnikov. 2019. S4L Self-Supervised Semi-Supervised Learning. In ICCV. 1476-1485.

[2] Ting Chen, Xiaohua Zhai, Marvin Ritter, Mario Lucic, and Neil Houlsby. 2019. Self-Supervised GANs via Auxiliary Rotation Loss. In CVPR. 12154-12163.

[3] Cheng Deng, Zhaojia Chen, Xianglong Liu, Xinbo Gao, and Dacheng Tao. 2019. Triplet-Based Deep Hashing Network for Cross-Modal Retrieval. CoRR abs/1904.02449 (2019).
[4] Jiuxiang Gu, Jianfei Cai, Shafiq R. Joty, Li Niu, and Gang Wang. 2018. Look, Imagine and Match: Improving Textual-Visual Cross-Modal Retrieval With Generative Models. In CVPR. 7181-7189.

[5] Dan Hendrycks, Mantas Mazeika, Saurav Kadavath, and Dawn Song. 2019. Using Self-Supervised Learning Can Improve Model Robustness and Uncertainty. In NeurIPS. 15637-15648.

[6] Mark J. Huiskes and Michael S. Lew. 2008. The MIR flickr retrieval evaluation. In ACM MIR. 39-43.

[7] Qing-Yuan Jiang and Wu-Jun Li. 2017. Deep Cross-Modal Hashing. In CVPR. $3270-3278$.

[8] Tsung-Yi Lin, Michael Maire, Serge J. Belongie, James Hays, Pietro Perona, Deva Ramanan, Piotr Dollár, and C. Lawrence Zitnick. 2014. Microsoft COCO: Common Objects in Context. In ECCV. 740-755.

[9] Yuxin Peng and Jinwei Qi. 2019. CM-GANs: Cross-modal Generative Adversarial Networks for Common Representation Learning. TOMM 15, 1 (2019), 22:1-22:24.

[10] Jose Costa Pereira, Emanuele Coviello, Gabriel Doyle, Nikhil Rasiwasia, Gert R. G. Lanckriet, Roger Levy, and Nuno Vasconcelos. 2014. On the Role of Correlation and Abstraction in Cross-Modal Multimedia Retrieval. IEEE TPAMI 36, 3 (2014), 521-535.

[11] Bokun Wang, Yang Yang, Xing Xu, Alan Hanjalic, and Heng Tao Shen. 2017. Adversarial Cross-Modal Retrieval. In ACM MM. 154-162.

[12] Bin Zhu, Chong-Wah Ngo, Jingjing Chen, and Yanbin Hao. 2019. R2GAN: CrossModal Recipe Retrieval With Generative Adversarial Network. In CVPR. 1147711486 